Reference:

Gabora, L. (2008). The cultural evolution of socially situated cognition. Cognitive Systems Research, 9(12), 104-113.

\title{
The Cultural Evolution of Socially Situated Cognition
}

\author{
LIANE GABORA \\ University of British Columbia
}

\author{
ADDRESS FOR CORRESPONDENCE: \\ Liane Gabora \\ Department of Psychology \\ University of British Columbia \\ Okanagan Campus, 3333 University Way \\ Kelowna, BC, V1V 1V7, CANADA \\ Email: liane.gabora[at]ubc.ca
}

\begin{abstract}
Because human cognition is creative and socially situated, knowledge accumulates, diffuses, and gets applied in new contexts, generating cultural analogs of phenomena observed in population genetics such as adaptation and drift. It is therefore commonly thought that elements of culture evolve through natural selection. However, natural selection was proposed to explain how change accumulates despite lack of inheritance of acquired traits, as occurs with template-mediated replication. It cannot accommodate a process with significant retention of acquired or horizontally (e.g. socially) transmitted traits. Moreover, elements of culture cannot be treated as discrete lineages because they constantly interact and influence one another. It is proposed that what evolves through culture is the mind; ideas and artifacts are merely reflections of its current evolved state. Interacting minds transform (in part) through through a nonDarwinian autopoietic process similar to that by which early life evolved, involving not survival of the fittest but actualization of their potential.
\end{abstract}

Key Words: acquired traits, autopoiesis, cultural evolution, natural selection, self-replication, social learning

\section{Introduction}

Other papers in this issue explore how cognition is shaped by the social matrix in which it is embedded. In this paper the reader is invited to take a step back and consider the dynamics that emerge when cognitive agents don just learn from one another, but put their own spin on what they learn, make sense of it in their own terms, and then express or implement their take on the ideas to others. Elements of culture start to create niches for one another. They become more complex with time, such that they might be thought of as constituting cultural lineages.

Some treat cultural change as merely a facet or dimension of biological evolution (Jablonka \& Lamb, 2006). ${ }^{[1]}$ Others, while not denying that culture has a dramatic impact on biological life, argue that culture constitutes a form of evolution in its own right, a second evolutionary process. On Earth the two 
are deeply intertwined (as illustrated by phenomena such as genetic assimilation, the Baldwin effect, and the fact that biological life had to come into being before culture could take hold). But in principle they need not be. For example, in a computer program in which artificial agents invent and imitate ideas for new gestures, but neither die nor give birth, there is still evolution (Gabora, 1995). It is not the agents physical form that is evolving, but their ideas. Fit gestures get imitated, and spread through the artificial society, while unfit gestures do not, such that over time the distribution of gestures implemented by agents becomes fitter. Indeed, cultural traits can be said to undergo descent with modification, and on the face of it cultural change is reminiscent of natural selection. It exhibits phenomena studied by population geneticists such as adaptation, punctuated equilibrium (Orsucci, in press), and drift (Bentley et al., 2004; Durham, 1991; Gabora, 1995), as well as features referred to by Mesoudi et al. (2004) as key

Darwinian properties including variation, competition, and inheritance. Cultural change is also cumulative; humans have a propensity to not just generate novelty but build on it cumulatively, adapting old ideas to new circumstances (the Ratchet effect). One individual modifies the basic idea of a cup by giving it a flat enough bottom to stay put when not in use, another adds a handle, making it easier to grasp, and yet another adds a spout, making it easier to pour from. Moreover, this cumulative change is adaptive. With each instantiation, the basic idea remains the same but the details change to make it more useful with respect to the prevailing situation or need. It is also complex and open-ended; there is no limit to the cultural novelty that can be generated.

This paper attempts to answer the question of whether the transmission and transformation of information across individuals occurs through a Darwinian process, and in what sense (if any) culture can rightly be said to evolve . We begin by examining why organisms do not inherit acquired characteristics, and how this impacts their evolution. This is key because acquired change is inherited in culture $^{[2]}$, though note that here inherited merely means transmitted or passed on without implying genetic mediation. To the extent that not inheriting acquired characteristics is central to how organisms evolve, answers from biology will not translate to culture. Noting that current origin of life theories suggest that the earliest life forms, referred to as protocells, also inherited acquired characteristics, we examine the hypothesis that the mechanisms by which culture evolves are more akin to those underlying the evolution of protocells than modern-day life.

\section{Is Culture Darwinian?}

It is often suggested that culture, or the creative ideas that propel it, evolve through a process that is Darwinian (e.g. Campbell, 1960; Cavalli-Sforza \& Feldman, 1981; Cloak, 1973; Dawkins, 1975; Plotkin, 1994; Maschner, 1996; Cziko, 1997, 1998; Spencer, 1997; Simonton, 1998, 1999a, b; Aunger, 2000; Mesoudi et al., 2004, 2006). Mesoudi et al. (2004) argue that because Darwin knew nothing of genes when he proposed his theory of natural selection, rejecting a Darwinian theory of culture on the grounds that we do not know what might be the cultural equivalent of genes is to apply a criterion so strict it would have led early biologists to reject the fundamental case made for evolution through natural selection in The Origin . But this is not the case, for Darwin s theory resolved a paradox that is nonexistent with respect to culture.

The paradox faced by Darwin and his contemporaries was to explain the following: how does change accumulate when acquired traits are lost? In most domains, change is retained, at least until another change overrides it. Once an asteroid has collided into a planet, for example, it does not revert back to the state of having not collided into a planet. But in biology there is a continual backtracking to an earlier state. Change arising during a lifetime, for example as a result of interaction with others, is obliterated at the end of each generation. A rat whose tail is cut off does not give birth to rats with cut 
tails. Thus the question that Darwin s theory answered was: if new modifications keep getting discarded, how is it that the form of living things has changed over time? The ingenuity of his approach was to look for change at the level of the population rather than the individual. Individuals who are better equipped to survive in their given environment leave more offspring (are selected $>$ ) and thus their traits are better represented in the next generation. Over generations this can lead to substantial change in the population of individuals as a whole.

The facts that inspired Darwin $>$ s theory of natural selection are unique to biology. Neither social scientists nor cognitive scientists are faced with a similar paradox. Cultural traits and artifacts do inherit acquired characteristics, so change resulting through creative thought processes, or interaction with peers or other elements of the environment, are retained. Once someone came along with the idea of putting a handle on a cup, cups with handles were here to stay. Inheritance of acquired traits allows for change that is orders of magnitude faster than change mediated by natural selection (for example, later today you may retell this argument in simplified form for a child). Thus we do not share the biologist $\mathrm{s}$ need to account for how change occurs despite a discarding of acquired traits each generation. It is therefore appropriate that Darwin solution not be embraced in a domain where the problem that solution was designed to solve does not exist.

Even if there is not the same pressing need to come up with a population-level mechanism of change as there was for biologists in Darwin $>$ s time, might not still the evolution of culture be described in terms of natural selection? ${ }^{[3]}$ This question no longer means what it would have meant in Darwin $\mathrm{s}$ time because the theory of natural selection has been rendered in precise mathematical terms, and for natural selection to be applicable, the following criteria must be met. First, there must be a population of self-replicating entities. Second, individuals must be lost from the population and replaced by new ones, giving rise to discreet or overlapping generations. Third, there must be competition for scarce resources. Fourth, there must be heritable random variation with respect to innate (genetically mediated) characteristics. Fifth, there must be no inheritance of acquired characteristics (or at least it must be negligible compared to change due to differential replication of individuals with heritable variation competing for scarce resources). In other words, natural selection cannot describe a process in which there is nothing prohibiting horizontal transmission from drowning out the effects of vertical transmission (or swamping the phylogenetic signal ). This last condition (no inheritance of acquired traits) makes the mathematical description of biological change tractable because one is simply dealing with changes in the frequencies of physically realized (i.e. already existing) entities, and variants of them generated through random mutation. Actually, in many biological situations these assumptions are not fully met (e.g. assortative mating), but they manage to hold as useful approximations and in some cases the equations can be modified to account for systematic biases. In biology one does not have to take into account potential variations to an entity that could come about through intuition, strategic thinking, or the myriad interactions it could have with the outside world. In social exchange, not only is acquired change inherited, but it comes about not through selection amongst competing randomly generated structures but through context-driven modification of existing structure. Orsucci (2002) refers to the set of cognitive states accessible from the current cognitive state as the adjacent possible . When an entity has the potential to change to many different states (given the various possible contexts it could interact with), we can say that it is in a potentiality state with respect to context (Gabora \& Aerts, 2005). ${ }^{[4]}$

Consider the following example of how context can affect a mind that is in a potentiality state. Let us say that Abe has the intension of determining whether Bess is a compatible mate. His mind is in a certain state that we label $p\left(t_{0}\right)$. He talks to her, likes her, and asks her out. Let us refer to the new state of mind in which this circumstance inspires this invitation, as $p_{1}\left(t_{l}\right)$. Now consider the same situation but this time he does not feel inspired to ask her out. Let us call the state of mind suggested by thissituation 
as $p_{2}\left(t_{1}\right)$. One cannot say that the state of mind $p\left(t_{0}\right)$ prior to talking to Bess had as a true proposition $\vartheta \mathrm{I}$ will ask her out However, one also cannot say that it had as a true proposition I will not ask her out Because of the contextual nature of the situation, neither of the propositions was true, but the state $p\left(t_{0}\right)$ had the potential for either to become true depending on the context. It isn $\mathrm{ta}$ one or the other is true situation, nor a situation of a competition amongst two pre-defined possibilities. It is because the truth of the proposition becomes actual in the process of being influenced by the context that the propositions formulated to describe this type of situation entail a nonclassical logic. The only way one can describe this with a classical formalism is to treat a set of potential, contextually elicited states of one entity as if they were actual states of a collection of entities, or possible states with no effect of context, even though the mathematical structure of the two situations is completely different.

Natural selection is inappropriate for the description of states of potentiality such as this. It can describe actual variation in a population of entities, but not potential variations of one entity. ${ }^{[5]}$ It is only possible to ignore the problem of incomplete knowledge of context if all contexts are equally likely, or if context has a temporary or limited effect. Because in biology, change accrued over the lifetime of an organism is obliterated at the end of each generation and not drawn into the lineage, the only contextual interactions with lasting impact are those that affect survival or the procurement of a mate. So in biology it has been possible to get away with ignoring the lack of knowledge of context problem and worry solely about the problem of lack of knowledge concerning the entity (where in selection theory the entity is a population of organisms), a problem that stochastic models such as those used in population genetics are equipped to handle. In culture, however, the inheritance of acquired traits makes the limitations of Darwinism more acute than for biology. Neither are all contexts equally likely, nor does context have a temporary or limited effect. So the assumptions that make natural selection a useful approximation do not hold.

\section{Inheritance of acquired characteristics}

That organisms do not inherit acquired characteristics is rather obvious, but the underlying reason for it is subtle, and stems from constraints on the kind of structure that is able to self-replicate. Analysis of the formal requirements for self-replication led von Neumann (1966) to postulate that a self-replicating automaton consists of coded information that gets used in two distinct ways. The first way is as a set of self-assembly instructions that are actively deciphered to construct a replicant. In this case, the code functions as interpreted information. The second way is as a self-description that is passively copied to the next replicant. In this case, the code functions as un-interpreted information. (To put it more loosely, the interpreting can be thought of as now we make a body and the un-interpreted use of the code as

$\checkmark$ now we make something that can itself make a body $>$.) Since biology is the field that inspired this distinction, naturally it applies here. DNA is copied — without interpretation — to produce gametes during meiosis. If gametes unite to form a germ cell, their DNA is decoded - interpreted - to synthesize the proteins necessary to assemble a body. There are of course deviations from this in present-day life, viruses being an oft-cited example, but these deviations can be accommodated within a Darwinian framework.

The most salient consequence of using a self-assembly code to replicate is that inheritance of acquired characteristics is (by and large) prohibited. What gets transmitted from parent to offspring in organisms is genetic self-assembly instructions, one set from each parent, and these instruction sets have not changed since they were formed through meiosis. So learned behavior and knowledge are not inherited by offspring. Thus biological organisms are shielded from change accrued during a lifetime. 
It has been suggested that the evolutionary character of culture reflects that cultural traits such as memes or artifacts are self-replicating automata, or replicators ${ }^{[6]}$ (Aunger, 2000; Lake, 1998). But even speaking loosely or metaphorically, an idea or artifact or meme cannot be said to possess a code that functions as both a passively copied self-description and a set of actively interpreted self-assembly instructions (Gabora, 2004). And accordingly, in culture acquired change is inherited. This is why the characteristics of artifacts can change considerably faster than the genomes of the individuals who produce them. Since viruses also replicate by way of a self-replication code (although not exclusively their own), they too are shielded by and large from inheritance of acquired traits. Thus epidemiological treatments of culture are similarly flawed (their infectiousness notwithstanding).

Lake (1998) argues that some but not all socially transmitted ideas and artifacts are replicators. The argument begins with a distinction between the expression versus the symbolically coded representation of cultural information. Whereas, for example, singing a song is an expression of a musical concept, a musical score is a representation of it. As another example, the spontaneous verbal explanation of an idea is an expression, whereas the text version of it is a representation. Lake comments that some cultural entities, such as village plans, constitute both a representation of a symbolic plan, and an expression of that plan, because they are both expressed by and transmitted through the same material form. However neither the expression nor the representation of a plan involves either interpreting or uninterpreted copying of a self-assembly code. A village plan does not, on its own, produce little copies of itself. A musical score does not generate offspring scores $\vartheta$. The perpetuation of structure and the presence of a symbolic code do not guarantee the presence of a replicator. Symbolic coding is not enough; it must be a coded representation of the self.

This distinction was recognized by Maturana and Varela (1980), who use the term allopoietic to describe an entity (such as a village plan) that generates another entity (such as a village) with an organization that is different from its own (e.g. while a village plan is a two-dimensional piece of paper, it generates something that is three dimensional and constructed of a variety of materials). Maturana and Varela contrast this with autopoietic, a term used to describe an entity composed of parts that regenerate themselves and thereby reconstitute the whole. It thus generates another entity with an organization that is nearly identical to its own. A self-replicating automaton is autopoietic. Socially transmitted elements of culture are not.

\section{Treating Units of Cultural Information as Separately Evolving Lineages}

The Darwinian view of culture has led to models that treat each unit of cultural information as a separately evolving lineage (Boyd \& Richerson, 1985; Cavalli-Sforza \& Feldman, 1981; Durham, 1991). These models examine the conditions under which such mutated cultural units pass vertically through a family, or horizontally through a community by imitation within an age cohort, and proliferate. To justify that this kind of Darwinian approach is viable even when there is nothing actually replicating, it was shown that a mathematical model of culture can exhibit replicator dynamics without any underlying selfreplicating structure (Henrich \& Boyd, 2002). On the basis of this it is claimed that cultural evolution proceeds without self-replication of any kind. The question, however, is not whether their model exhibits replicator dynamics (a concept that does not capture the complex, adaptive, open-ended nature of even biological evolutionary change). The question is does it exhibit cultural dynamics? Is it coming up with things like hopscotch and wedding vows?

Kauffman (1999) argues that this kind of approach is impoverished, in part because it suffers from the inability to account for the source of new cultural forms. As he puts it A rich web of conceptual interactions is at work as humans happen upon, design, and implement a combinatorially exploding diversity of new goods and services. This web structure of technological and cultural evolution is far richer, and far closer to the truth, than mere meme descent with modification. $\rightarrow$ It has been shown 
that the mind possesses the kind of small-world structure that characterizes many complex systems (Schilling, 2005). The description of culture and the minds that constitute it as web-like echoes current descriptions of the earliest forms of life, and he and others have provided an impressive body of theoretical and empirical evidence that this kind of self-organized, web-like structure is essential to the capacity to evolve (Kauffman, 1993). In a similar vein, Maturana and Varela (1980) argue that autopoietic organization is the defining characteristic of life — and indeed what makes a structure evolvable What autopoietic organization offers is the potential for a self-sustained stream of interacting and combining, resulting in new forms with new dynamics. The autopoietic organization of the human mind arises from the associative structure of memory. The fruits of associative processes (e.g. analogy, concept combination, divergent thinking) serve as ingredients for analytic processes (e.g. logic, symbol manipulation, formal operations), and vice versa (Gabora, 2000, 2002, 2003). The upshot is an ability to internalize the world (in terms of both its causal and its correlational structure), and an unprecedented capacity for creativity.

Treating one kind of cultural information in isolation from others is akin to removing organs from a dissected animal one by one and hoping to understand each in isolation from the others and the organism at large. It is reductionist. Ideas may take seemingly static forms in the physical world such as works of art or books of government policy. But in the minds of those who behold them they are charged with the potential to dynamically interact with goals, plans, schemas, desires, attitudes, fantasies, and unborn ideas, and it is through these interactions that their meaning and significance are derived. Very often this happens in a social milieu, each individual providing the idea with a different context, a different ecology of mind in which to be understood, and therefore able to flesh it out or give it a fresh twist. In this way, different domains of human culture influence, cross-fertilize, or contaminate one another to such an extent that it is misleading to consider them separate lineages. In short, the genre of model that treats cultural information as constituting separate lineages evolving through Darwinian processes, and that does not take the self-replication issue seriously, fails to incorporate the smallworld , autopoietic structure of the mind, which is essential to its capacity to perpetually see one thing in terms of another and thereby adapt, invent, and revise. These models may exhibit cumulative change of transmitted traits in the same sense that a each time a tape is copied it accumulates more distortion. But they are not able to generate novelty in a manner that is anything like human creativity, and therefore can have little bearing on the observed richness of human culture.

\section{Self-replication without a self-assembly code}

If organisms evolve because they are self-replicating automata, and socially transmitted elements of culture are not self-replicating automata, must it be concluded that culture does not evolve? No, because, von Neumann self-replicating automata are not the only kind of self-replicating structure. Let us investigate a second, more primitive kind, and then see if there is something in culture that possesses it. Examining the earliest stages in the evolution of organic life can constrain the development of a realistic theory of the evolution of culture. Thus the topic of how life began is not a detour but a key step forward to a deeper understanding of evolution in general and culture in particular.

Present day life replicates using a template, a coded set of instructions encoded in DNA or RNA for how to make a copy of itself. The probability of such a structure arising spontaneously is exceedingly small; Hoyle infamously compared it to the probability that a tornado blowing through a junkyard would assemble a Boeing 747 (Hoyle, 1981). The implausibility of the spontaneous appearance of a selfassembly code has led to skepticism toward replication-first theories of the origin of life, and the widespread acceptance of metabolism-first theories. According to the metabolism-first view, life began with a chemically isolated, self-organized set of simple collectively replicating molecules - for example through 
autocatalytic closure $^{[7]}$ of catalytic polymers - and genetically mediated template replication came afterward (Bollobas, 2001; Bollobas \& Rasmussen, 1989; Dyson, 1982, 1985; Gabora, 2006; Kauffman, 1986, 1993; Morowitz, 1992; W echtersh euser, 1992; Weber, 1998, 2000; Williams \& Frausto da Silva, 1999, 2002; Vetsigian et al., 2006). Polymers catalyze reactions that generate other polymers, increasing their joint complexity, until together as a whole they form a protocell that can more or less replicate itself. Due to criticality and threshold phenomena in random graphs, though the number of different polymers increases exponentially, the number of reactions by which they can interconvert increases faster than their total number (Cohen, 1988; Erd $>\mathrm{s} \& \mathrm{R}$ nyi, 1960). Thus, as their diversity increases, so does the probability that some subset of the total reaches a critical threshold where there is a catalytic pathway to every member. The set may be said to be autocatalytically closed because although no polymer catalyzes its own replication, each catalyzes the replication of another member of the set. So long as each polymer is getting duplicated somewhere in the set, eventually multiple copies of all polymers exist.

At least some subset of the polymers spontaneously adhere to one another, forming a spherical vesicle that encloses the polymer set. Such a structure is prone to fission or budding, where part of the vesicle pinches off and it divides in two. Replication is far from perfect, thus offspring are unlikely

to be identical to parent . But so long as there is at least one copy of each polymer in each of the two resulting vesicles, they can self-replicate, and continue to do so indefinitely, or until their structure changes drastically enough that self-replication capacity breaks down. Replication is sloppier and more haphazard than a von Neumann self-replicating automaton, but nonetheless gets the job done.

Note that the resulting structure is autopoietic because the parts collectively reconstitute the whole, but it happens in a piecemeal manner, through bottom-up interactions rather than top-down interpretation of a genetic code. Therefore replication occurs with low fidelity, and there is nothing to prohibit the inheritance of acquired characteristics. A change to one polymer persists after fission occurs, and this could cause other changes that have a significant effect on the lineage further downstream. Evolution of these early life forms has been described as a non-Darwinian process taking place through horizontal exchange (i.e. not restricted to vertical transmission from parent to offspring) of innovationsharing protocols (Vetsigian et al., 2006). It was not until the genetic code came along - and the process in which self-assembly instructions are copied (meiosis) became distinct from developmental processes - that acquired characteristics could no longer be passed on to the next generation (Gabora, 2006).

Thus we have two kinds of self-replication (Gabora, 2004). Coded self-replication, such as is seen in present-day organisms, uses self-assembly instructions as proposed by von Neumann. This ensures they replicate with high fidelity, and acquired characteristics are not inherited. Uncoded selfreplication, such as is seen in protocells, involves autopoiesis. This is a low fidelity means of replication, and there is nothing to prohibit inheritance of acquired characteristics. Note that it is often said that because acquired traits are inherited in culture, culture cannot be described in evolutionary terms. It is ironic that this is also true of the earliest stage of biological life itself.

\section{Does culture evolve in the same sense as early life?}

The next question is: might elements of culture possess the same kind of autopoietic structure as a protocell, which replicates (albeit haphazardly) without a self-assembly code? The answer is again no. Neither a frisbee nor a business contract, nor the ideas behind them, consist of parts that catalyze or evoke or in some other way regenerate one another such that together they form an integrated whole that can reconstitute itself. The transmission of an idea is more akin to the transmission of a radio signal and its 
reception by one or more radios; neither an idea nor a radio signal self-replicates, not in the von Neumann sense of present-day life, nor in the autocatalytic sense of early life.

Another possibility is that autopoietic structure does exist in culture, but it is not the cultural trait, nor the idea behind it, but the web of interrelated ideas that constitutes an individual $\mathrm{s}$ internal model of the world. It has been suggested that much as the autocatalytic set is an uncoded self-replicating structure that brought about biological evolution, the human mind is an uncoded replicator that brought about cultural evolution (Gabora, 1998, 2000, 2007b). Intimations of this theory can be found in diverse sources. Fleissner and Hofkirchner (1996) describe cognition as a process of self-organization cycles and cultural systems as self-structuring, self-reproducing, and self-creating. Perhaps the first to think in such terms was Geiger (1985) who writes Culture is argued to involve ecological interactions exhibiting analogies to the interaction of chemical species in autocatalytic biomolecular reactions. In a similar vein, Sereno (1991) proposes that a common problem was solved by the origin of life and the origin of thought:

a unique single-celled symbolic representational system first arose from a pre-biotic chemical substrate at the origin of life, permitting Darwinian evolution to occur. A similar autonomous symbolicrepresentational system did not emerge on any intermediate level until the origin of thought and language from the substrate of prelinguistic neural activity patterns in the brains of Pleistocene hominids.

We start by noting that a socially situated human memory is sparse, distributed, and contentaddressable (Hinton, McClelland, \& Rummelhart, 1986; Hopfield, 1982; Kanerva, 1988; Lin et al., 2006). Thus elements of culture are not stored in memory as discrete chunks (Goldman-Rakie, 1992; Miyake \& Shah, 1999) but overlap, and as such are woven into a flexible, integrated model of how different aspects of the world relate to one another, and how to make ones way in it: a worldview. The distributed nature of the memory structure in which a worldview is encoded is what enables us to reason about one situation in terms of another, adapt ideas to new circumstances or different audiences, evaluate new experiences in terms of previous ones, and blend information from different domains, as in a pun or double entendre or the construction of an item that has both a useful and a decorative function (Gabora, 2007c). All these depend on the ability to forge an association between two things and thereby see one through the lens of the other. But this leads to a chicken-and-egg paradox. Until memories are woven into a worldview, how can they generate the remindings and associations that constitute a stream of thought? Conversely, until a mind can generate streams of thought, how does it integrate memories into a worldview? How could something composed of complex, mutually dependent parts come to be?

The proposal that it comes about through a process referred to as conceptual closure follows straightforwardly from the concepts of autopoiesis and closure. Applying them to cognition, just as polymers catalyze reactions that generate other polymers, retrieval of an episode or other item from memory evokes another, which evokes yet another, thereby forging new associative paths. In a distributed memory, this increases the probability that episodes connected by a dense web of associative paths start to get treated as instances of a particular concept (Rummelhart \& McClelland, 1985). Concepts facilitate streams of thought, which forge connections between more distantly related clusters. The ratio of associative paths to concepts increases until (due to the threshold effect discussed in the origin of life context) it becomes almost inevitable that a giant cluster emerges. When for any one item in memory there exists a possible associative path to any other, they form an integrated conceptual web, that can be described by a connected closure space.

The theory that cultural evolution is mediated by the uncoded replication of worldviews speaks to a concern raised by Boone and Smith (1998: S142-3) and others that In adopting the Darwinian framework, evolutionary archaeologists have simply substituted phenotypic variation for genetic. With uncoded replication, the genotype/phenotype distinction has no apt equivalent. Since the mode of change is not template-driven, there is no portion of an artifact that is (like a genome) shielded from acquired change. And there is no portion that is (like a body) shed at the end of a generation. The mistake of 
evolutionary archaeologists and other cultural Darwinists may not be the notion that artifacts evolve, but that they evolve the way present-day life evolves. Their evolution may be more akin to the evolution of these earliest forms of life, and the unit of replication the web of memories and knowledge that constitutes a worldview.

The idea then is that the self-replicating structures in culture are not memes but minds, or more accurately, worldviews. A worldview is not the coded sort identified by von Neumann; it is uncoded, like the autocatalytic sets postulated to be the earliest forms of life. A worldview replicates not all-at-once but in a piecemeal manner through social learning via the exchange of knowledge, stories, perspectives, actions, artifacts, and so forth. Worldviews are therefore not so much replaced by as transformed into (literally) more evolved ones. The current evolutionary state of a worldview is reflected in the things one does, says, or makes; different situations expose different facets of a worldview (like cutting a fruit at different angles exposes different parts of its interior). As with protocells in metabolism-first origin of life scenarios, the process is bottom-up rather than top-down (dictated by selfassembly instructions), and therefore characteristics acquired over a lifetime are passed on.

The proposal that the worldview evolves as a unit is consistent with the view that the contents of the mind are interactive (Aerts \& Gabora, 2005a,b; Bickhard, 2000; Gabora \& Aerts, 2002; Gabora, Aerts, \& Rosch, in press; Rosch, 1999). That is, they are not fixed until elicited by a context, and it is contextual interaction that causes the relevant representations to take on a well-defined form. The social self can be said to emerge through the assimilation of norms, values, and conventions over the course of this interactive process (Bickhard, 2004). This brings us to a version of the extended mind view in which elements of culture are not duplicated across minds but assimilated into different autopoietic structures where their potential to interact and provide meaning is differently realized.

\section{What Brought About the Capacity for Complex Culture?}

Many have noted that cultural evolution is cumulative. It has been suggested that this alone differentiates human culture from that of other species (Boyd \& Richerson, 1996). Cumulative change is exhibited by a computer model of cultural evolution that uses conceptual structure to generate novelty (Gabora, 1995), but also by a model in which change accrues through mere copying error (Eerkins \& Lipo, 2005). Indeed cumulative change is relatively easy to come by; in the days of taping music, each time the tape was copied it became cumulatively more scratched. This suggests that it is not just because human cultural evolution is cumulative that it is distinctive. It is distinctive because of what accumulates, and what accumulates is change that is complex, adaptive, and open-ended because it is born in a conceptual network powered by both associative thought (which detects relationships of correlation) and strategic thought (which detects relationships of causation). Theories of culture based on the view that what makes human culture distinctive is that it is cumulative do not incorporate concepts and how they are related, let alone organized into an internal model of the world, or worldview. Nor do they incorporate how a worldview constrains and stimulates how novelty is generated through exploring not just what is but what could be . Efforts to build psychology into such models are limited to phenomena such as copying error (where cultural information is distorted as it passes from one individual to another) or conformist bias (a tendency to conform to what others are doing). But copying error and conformist bias hardly move us further along the path toward a theory that can account for the emergence of even simple acts of conceptual transfer such as the invention of marshmallows shaped like Smurfs let alone complex, collaborative manifestations of culture such as the production of Star Wars.

The view that what makes human culture distinctive is merely that it is cumulative often goes hand in hand with the view that human cultural evolution arose through enhanced capacity for imitation. There are several versions of this; one of the more provocative is that proposed by Merlin Donald (1993). He argues that approximately two million years ago the mind underwent a transition from an episodic mode of cognitive functioning, which is more or less stuck in the here and now to a 
uniquely human mimetic mode, characterized by the capacity for mime, imitation, gesture, and the rehearsal of skill. A problem with this proposal is that imitation is commonplace in other species, as are the mirror neurons that underlie imitative skill (Arbib, 2002). Moreover, the others - mime, gesture, and skill rehearsal - rely on what Karmiloff-Smith (1992) refers to as representational redescription (RR): the capacity to recursively operate on or manipulate the contents of thought and thereby refine an idea or motor act, or retrieve an event from the past through the linking of associations. Moreover, imitation, though possible without RR, would be enhanced by it; it would enable imitated skills to be reworked and perfected, perhaps one step at a time. Thus it seems more parsimonious to propose that the transition was due to the onset of RR, which enabled mime, gesture, and skill rehearsal, and merely enhanced imitation.

This simultaneously alleviates another problem with Donald $>$ s account: the idea that enhanced imitative capacity enabled us to become more firmly tethered in a cultural network, which was critical to the evolution of our distinctiveness. In fact as a species we exhibit the opposite tendency, to go our own way and do our own thing, and creative individuals, those who have the most transformative effect on culture, are the least tethered of all, with strong leanings toward isolation, nonconformity, rebelliousness, and unconventionality (Crutchfield, 1962; Griffin \& McDermott, 1998; Sulloway, 1996). Thus the claim that imitative capacity is what gave rise to human culture is problematic.

The alternative developed here is that increased brain size allowed for more fine-grained encoding of memories and thus denser associations amongst them, enabling the onset of representational redescription, abstract thought, and thereby conceptual closure. Note that it is not the presence of but the capacity for an integrated worldview that the human species came to possess. An infant may be born predisposed toward conceptual integration and the formation of an internal model of the world and how its parts relate. But the process must begin anew in each young mind. The resulting cognitive structure is autopoietic in that one memory can evoke or regenerate another, which regenerates another, and so forth. The propensity for this kind of regenerative thought lends a self-mending quality to the worldview. When one encounters a situation that does not fit or make sense with respect to ones view of the world, thought and dialogue function to weave stories that mend conceptual gaps and thereby reconstitute ones worldview (Gabora, 1999). The term natural selection not apply to this process, nor does Campbell s (1960) phrase blind variation and selective retention generated strategically rather than blind $>$, but because new ideas (and revisions to these ideas) are evaluated sequentially, and this evaluation can itself change the state space and/or fitness function, and consequently no two variants are ever evaluated according to the same selection criterion (Gabora, 2005, 2007a). Thus the development of creative ideas is not a matter of selecting but honing

\section{Summary and conclusions}

The human capacity to exchange and build on one another s ideas has completely transformed this planet. It is tempting to describe the process of cultural change in Darwinian terms, in part because (1) the parallels between biology and culture are striking, and (2) Darwin s theory had a profoundly unifying effect on biology. Indeed Mesoudi et al. (2004) rightly assert that culture exhibits key Darwinian properties $\vartheta$. But having these properties does not imply that the mechanisms are similar, and they are not; indeed the paradox that necessitated the theory of natural selection in biology does not exist with respect to culture. Early biologists were motivated by the need to explain how change accumulates in a domain where acquired traits are not retained at the individual level. Selection theory was accordingly designed to describe descent with modification at the population level, in organisms that do not inherit acquired characteristics because they are von Neumann self-replicating automata. Self-replicating 
automata use a self-assembly code that is both actively transcribed to produce a new individual, and passively copied to ensure that the new individual can itself reproduce. Cultural traits and artifacts are not self-replicating automata, even those such as a village plan that contain a code, because the code consists of instructions for how to generate something else, not a copy of itself. They are allopoietic, not autopoietic. Cultural traits do inherit acquired traits - the effects of creative modification or contextual interaction with the environment are retained. So cultural theorists do not share the biologists need to explain how change occurs in the face of periodic reversion to a previous state. Their challenge is to describe cognitive states that involve potentiality, and that change through interaction with an incompletely specified context, as occurs in (for example) dialogue or play.

It is sometimes said that culture evolves without any sort of self-replicating structure, or that since the kind of modification with descent exhibited in culture cannot be described by natural selection, there is no theory of evolution that can explain cultural change (e.g. Fracchia \& Lewontin, 1999). It does seem unlikely that culture involves von Neumann self-replicating automata. But natural selection of selfreplicating automata is not the only means by which evolution can occur. Indeed another sort of process was necessary to get life to the point where it replicated using a self-assembly code and was thereby able to evolve through natural selection. Current widely held theories about the origin of life hold that protocells, the earliest forms of life, evolved through a non-Darwinian process. They self-replicated without a self-assembly code through autopoiesis, i.e. mutual regeneration of parts to reconstitute a new whole. Because uncoded self-replicating structures can inherit traits acquired between replication events, they may either survive intact or go extinct, or do something in between these two extremes: they can transform through the horizontal exchange of innovation.

We found a candidate for this more primitive, uncoded kind of self-replicating structure in culture: the worldview. It is posited that humans achieved this kind of integrated internal model of the world in virtue of the large cranial capacity and resulting dense associative structure of human memory. The mind generates cultural novelty not by selecting amongst predefined randomly-generated alternatives but by thinking through how it could work, and its capacity to shift between analytic and associative thought processes makes it ingeniously suited to this kind of mulling over $>$. Associative thought often proceeds through interaction with a context: a fortuitous encounter with a triggering stimulus, talking something over, or simply re-assessing where one is going based on the effects of ones actions. Natural selection cannot account for this kind of context-driven change because it cannot incorporate situations in which a product of culture could take many different forms depending on the worldviews of those creating it, and the circumstances under which they came together and interacted when they did. Consequently, it cannot account for the birth of new cultural traits except as the result of trial and error; moreover it cannot account for novelty that has new properties or that deviates in any substantial way from what has come before. It is proposed that worldviews evolve not through survival of the fittest but through transformation; they neither die nor survive intact but transform over generations as elements get incorporated and adapted to new circumstances. We cannot examine the evolution of worldviews directly, but we can do so indirectly, by examining how art, science, technology, media, social structures, and governing bodies are transforming the planet that sustains us.

\section{Acknowledgements}

The author acknowledges the support of a research grant from Foundation for the Future.

\section{REFERENCES}

Arbib, M. A. (2002). The mirror system: Imitation and the evolution of language. In C. Nehaniv and K. Dautenhahn (eds.) Imitation in animals and artifacts. Cambridge, MA: MIT Press.

Aunger, R. (2000). Darwinizing culture. Oxford: Oxford University Press.

Bamforth, D. B. (2002). Evolution and metaphor in evolutionary archaeology. American Antiquity, $67,435-52$. 
Barkow, J., Cosmides, L., \& Tooby, J. (1992). The adapted mind. New York: Oxford University Press.

Bentley, R. A., Hahn, M. W., \& Shennan, S. J. (2004). Random drift and culture change. Proceedings of the Royal Society: Biology, 271, 1443-1450.

Bickhard, M. H. (2000). Information and representation in autonomous agents. Cognitive Systems Research, 1, 65-75.

Bickhard, M. H. (2004). The social ontology of persons. In J. Carpendale \& U. Mueller (Eds.) Social interaction and the development of knowledge. Hillsdale, NJ: Erlbaum.

Buller, D. J. (2005). Adapting minds. Cambridge: MIT Press.

Buss, D. M. (1999/2004). Evolutionary psychology: The new science of the mind. Boston: Pearson.

Bollobas, B. (2001). Random graphs. Cambridge: Cambridge University Press.

Bollobas, B., \& Rasmussen, S. (1989). First cycles in random directed graph processes. Discrete Mathematics, 75(1-3), 55-68.

Boone, J. L., \& Smith, E. A. (1998). Is it evolution yet? A critique of evolutionary archaeology, Current Anthropology, 39, S141-73.

Campbell, D. T. (1960). Blind variation and selective retention in creative thought as in other knowledge processes. Psychogical Review, 67, 380-400.

Cavalli-Sforza, L. L., \& Feldman, M. W. (1981). Cultural transmission and evolution: A quantitative approach. Princeton: Princeton University Press.

Cloak, F. T. Jr. (1975). Is a cultural ethology possible? Human Ecology, 3, 161-182.

Cohen, J. E. (1988). Threshold phenomena in random structures. Discrete Applied Mathematics, $75,55-68$.

Crutchfield, R. S. (1962). Conformity and creative thinking. In H. G. Gruber, G. Terell, \& M. Wertheimer (Eds.) Contemporary Approaches to Creative Thinking. New York: Atherton.

Csanyi, V. (1989). Evolutionary systems and society: A general theory of life, mind and culture. Durham: Duke University Press.

Cziko, G. (1997). Without miracles: Universal selection theory and the second Darwinian revolution. Cambridge, MA: MIT Press.

Cziko, G. (1998). From blind to creative: In defense of Donald Campbell s selectionist theory of human creativity. Journal of Creative Behavior, 32(3), 192-212.

Dawkins, R. (1975). The selfish gene. Oxford: Oxford University Press.

Dasgupta, S. (2004). Is creativity a Darwinian process? Creativity Research Journal, 16(4), 403-413.

Durham, W. (1991). Coevolution: Genes, culture, and human diversity. Stanford: Stanford University Press.

Dyson, F. (1982). A model for the origin of life. Journal of Molecular Evolution, 18, 344-350.

Dyson, F. (1985/1999). Origins of Life. Cambridge University Press, Cambridge.

Eerkens, J. \& Lipo, C. P. (2005). Cultural transmission, copying errors, and the generation of variation in material culture and the archaeological record. Journal of Anthropological Archaeology, 24, 316-334.

Erd s, P., R nyi, A. (1960). On the evolution of random graphs. Publication of the Mathematical Institute of the Hungarian Academy of Sciences, 5, 17-61.

Fleissner, P., \& Hofkirchner, W. (1996). Emergent information. Biosystems, 2-3(38), 243-248.

Fracchia, J., \& Lewontin, R. C. (1999). Does culture evolve? History and Theory, 38, 52-78.

Foulis, D., \& Randall, C. (1981). What are quantum logics and what ought they to be? In: Beltrametti, E., \& van Fraassen, B. (Eds.) Current Issues in Quantum Logic 35. New York: Plenum.

Gabora, L. (1995). Meme and variations: A computer model of cultural evolution. In Nadel, L., \& Stein, D. (Eds.), Lectures in complex systems (pp. 471-486). Reading MA: Addison-Wesley.

Gabora, L. (1998). A tentative scenario for the origin of culture. Psycoloquy, 9(67).

Gabora, L. (1999). Weaving, bending, patching, mending the fabric of reality: A cognitive science perspective on worldview inconsistency. Foundations of Science, 3(2), 395-428. 
Gabora, L. (2000). Conceptual closure: Weaving memories into an interconnected worldview. In J. L. R. Chandler, \& G. Van de Vijver (Eds.) Closure: Emergent organizations and their dynamics (pp. 42-53). New York: Annals of the New York Academy of Science.

Gabora, L. (2002). Cognitive mechanisms underlying the creative process. In T. Hewett \& T. Kavanagh (Eds.) Proceedings of the fourth international conference on creativity and cognition. October 13-16, Loughborough University, UK, pp. 126-133.

Gabora, L. (2004). Ideas are not replicators but minds are. Biology \& Philosophy, 19(1), 127-143.

Gabora, L. (2005). Creative thought as a non-Darwinian evolutionary process. Journal of Creative Behavior, 39(4), 65-87.

Gabora, L. (2006a). Self-other organization: Why early life did not evolve through natural selection. Journal of Theoretical Biology, 241(3), 443-450.

Gabora, L. (2006b). The fate of evolutionary archaeology: Survival or extinction? World Archaeology, 38(4), 690-696.

Gabora, L. (2007a). Why the creative process is not Darwinian. Commentary on 'The creative process in Picasso's Guernica sketches: Monotonic improvements versus nonmonotonic variants' by D. K. Simonton. Creativity Research Journal. www.vub.ac.be/CLEA/liane/Reviews/guernica.htm

Gabora, L. (2007b). Mind: What archaeology can tell us about the origins of human cognition. In A. Bentley \& H. Maschner (Eds.). Handbook of theories and methods in archaeology. Walnut Creek CA: Altamira Press.

Gabora, L. (2007c). Revenge of the neurds $>$ : Characterizing creative thought in terms of the structure and dynamics of memory. Creativity Research Journal.

Gabora, L., \& Aerts, D. (2005a). Distilling the essence of an evolutionary process, and implications for a formal description of culture. In: Kistler, W. (Ed.) Proceedings of center for human evolution workshop \#5: Cultural evolution, May, 2000. Seattle: Foundation for the Future.

Gabora, L., \& Aerts, D. (2005b). Evolution as context-driven actualization of potential: Toward an interdisciplinary theory of change of state. Interdisciplinary Science Reviews, 30(1), 69-88.

Gabora, L., Rosch, E. \& Aerts, D. (2007). Toward an ecological theory of concepts. Ecological Psychology.

Geiger, G. (1985). Autocatalysis in cultural ecology: model ecosystems and the dynamics of biocultural evolution. Biosystems, 17(3), 259-272.

Griffin, M. \& McDermott, M. R. (1998). Exploring a tripartite relationship between rebelliousness, openness to experience, and creativity. Social Behavior and Personality, 26, 347-356.

Henrich, J., \& Boyd, R. (2001). Why people punish defectors: Weak conformist transmission can stabilize costly enforcement of norms in cooperative dilemmas. Journal of Theoretical Biology, 208, 78-89.

Henrich, J., \& Boyd, R. (2002). On modeling cognition and culture: Why replicators are not necessary for cultural evolution. Culture and Cognition, 2(2), 67-112.

Holliday, R. (1990). DNA methylation and epigenetic inheritance. Philosophical Transactions of the Royal Society of London B: Biological Science, 326(1235), 329-338.

Hoyle, F. (1981). Hoyle on evolution. Nature, 294, 105.

Karmiloff-Smith, A. (1992). Beyond modularity: A developmental perspective on cognitive science, MIT Press.

Kauffman, S. (1993). Origins of order. Oxford University Press, New York.

Kauffman, S. (1986). Autocatalytic sets of proteins. Journal of Theoretical Biology, 119, 1-24.

Kauffman, S. (1999). Darwinism, neoDarwinism, and the autocatalytic model of culture: Commentary on Origin of Culture, Psycoloquy, 10(22), 1-4.

Kolmogorov, A.N. (1933). Grundbegriffe der wahrscheinlichkeitrechnung. Berlin: Ergebnisse der Mathematik. 
Lake, M. (1998). Digging for memes: The role of material objects in cultural evolution. InRenfrew, C., Scarre, C. (Eds.), Cognition and material culture: The archeology of symbolic storage (pp. 77-88). London: McDonald Institute Monographs.

Maschner, H. D. G., Ed. (1996). Darwinian archaeologies. New York: Plenum Press.

Mayr, E. (1996). What Is a Species, and What Is Not? Philosophy of Science, 63(2), 262-277.

Mesoudi, A., Whiten, A., \& Laland, K. (2004). Toward a unified science of cultural evolution. Evolution 58(1), 1-11.

Mesoudi, A., Whiten, A., \& Laland, K. (2006). Toward a unified science of cultural evolution. Behavioral and Brain Sciences, 29,329-383.

Maturana, H. R., \& Varela, F. J. (1980). Autopoiesis and cognition: The realization of the living. Boston: Reidel.

Morowitz, H. J. (2002). The emergence of everything: How the world became complex. New York: Oxford University Press.

Newman S. A., \& M ller, G. B. (1999). Morphological evolution: Epigenetic mechanisms. In: Embryonic encyclopedia of life sciences. London: Nature Publishing Group.

Orsucci, F. (2002). Changing mind: Transitions in natural and artificial environments. London: World Scientific.

Orsucci, F. (in press). The complex coevolution of information technology ecosystems. Hershey, NY: Idea Books.

Pinker, S. (1995). The language instinct. HarperPerrenial.

Pitowsky I. (1989). Quantum Probability, Quantum Logic, Lecture Notes in Physics 321, Springer, Berlin.

Plotkin, H. (1994). Darwin machines and the nature of knowledge. Boston: Harvard University Press.

Pocklington, R., \& Best, M. L. (1997). Cultural evolution and units of selection in replicating text. Journal of Theoretical Biology, 188, 79-87.

Randall, C., \& Foulis, D. (1976). A mathematical setting for inductive reasoning. In C. Hooker (Ed.) Foundations of probability theory, statistical inference, and statistical theories of science III. Dordrecht, The Netherlands: Reidel.

Rummelhart, D. E. \& McClelland, J. L. (1985). Parallel distributed processing: Explorations in the microstructure of cognition.Cambridge MA: MIT Press.

Schilling, M. (2005). A small-world network model of cognitive insight. Creativity Research Journal, 17, 131-154.

Schwartz, J. H. (1999). Sudden origins. New York: Wiley.

Sereno, M. (1991). Four analogies between biological and cultural/linguistic evolution. Journal of Theoretical Biology, 151, 567-507.

Simonton, D. K. (1998). Donald Campbell $>$ s model of the creative process: Creativity as blind variation and selective retention. Journal of Creative Behavior, 32(3), 153-158.

Simonton, D. K. (1999a). Origins of genius: Darwinian perspectives on creativity. New York: Oxford.

Simonton, D. K. (1999b). Creativity as blind variation and selective retention: Is the creative process Darwinian? Psychological Inquiry, 10, 309-328.

Spencer, C. S. (1997). Evolutionary approaches in archaeology. Journal of Archaeolical Research, 5, 209-264.

Sulloway, F. (1996). Born to Rebel. New York: Pantheon.

Vetsigian, K., Woese, C., Goldenfeld, N. (2006). Collective evolution and the genetic code. Proceedings of the New York Academy of Science USA, 103, 10696-10701.

Von Neumann, J. (1966). The theory of self-reproducing automata. Chicago: University of Illinois Press.

W chtersh user, G. (1992). Groundwork for an evolutionary biochemistry: the iron-sulfur world. Progress in Biophysics and Molecular Biology, 58, 85-201. 
Weber, B. H. (1998). Emergence of life and biological selection from the perspective of complex systems dynamics. In G. van de Vijver, S. N. Salthe, \& M. Delpos (Eds). Evolutionary Systems:

Biological and epistemological perspectives on selection and self-organization. Dordrecht: Kluwer.

Weber, B. H. (2000). Closure in the emergence and evolution of life: Multiple discourses or one? In J. L. R. Chandler, \& G. Van de Vijver (Eds.), Closure: Emergent organizations and their dynamics, (pp. 132-138). New York: Annals of the New York Academy of Science.

Williams, R. J. P., \& Frausto da Silva, J. J. R. (1999). Bringing chemistry to life: From matter to man. Oxford: Oxford University Press.

Williams, R. J. P., \& Frausto da Silva, J. J. R. (2002). The systems approach to evolution. Biochemical and Biophysical Research Communications, 297, 689-699.

${ }^{11}$ This paper does not address the issue of the extent to which the human capacity for language and other elements of culture is genetically assimilated and/or part of the human phenotype, which is discussed at length elsewhere (e.g. Barkow et al., 1992; Buss, 1999/2004; Buller, 2006; Gabora, 2006; Pinker 1995).

${ }^{[2]}$ Culture is thus sometimes referred to as Lamarckian.

${ }^{[3]}$ It is worthwhile pointing out that neo-Darwinism, powerful though it is, does not begin to provide a comprehensive account of biology (e.g. Holliday, 1990; Kauffman, 1993; Newman and M ller, 1999; Schwartz, 1999) let alone culture. It cannot account for situations involving assortative mating, symbiosis, epigenetic or selforganized processes such as the origin of life discussed earlier, nor the appearance of new forms with new characteristics, particularly if they emerge through contextual processes such as interaction with the environment. ${ }^{[4]}$ Potentiality and contextuality both stem from the fact that we inevitably have incomplete knowledge of the universe in which an entity is operating. We proceed as if a process can be isolated from its context and as if there could exist a class of processes that are identical, though it is obvious that any process and its context when considered in complete detail is a unique event. When the state of the entity of interest and/or context are in constant flux, or undergoing change at a resolution below that which we can detect but nevertheless affect what emerges at the entity-context interface, this gives rise in a natural way to nondeterministic change. In reality the universe is so complex we can never describe with complete certainty and accuracy the context to which an entity is exposed, and how it interacts with the entity. There is always some possibility of even very unlikely outcomes. However, there are situations in which we can predict the values of relevant variables with sufficient accuracy that we may consider the entity to be in a particular state, and other situations in which there is enough uncertainty to necessitate the concept of potentiality. Thus a theory that concerns how an entity undergoes a change of state must take into account the degree of knowledge we as observers have about the context.

${ }^{[5]}$ More generally, when the entity of interest is a set of multiple physically realized or actual (as opposed to potential) sub-entities from amongst which some subset is selected, this is a deterministic process that can be described by a classical probability theory such as the mathematical theory of natural selection. Selection theory is concerned only with factors internal to the population, so nondeterminism reflects a lack of knowledge of the state of the entity (the entity being not any one particular organism but the population). Nondeterminism that arises through lack of knowledge concerning the state of the entity can be described by classical stochastic models (e.g. Markov processes) because the probability structure is Kolmogorovian ( $\mathrm{i}$ the mathematical theory of natural selection. Selection theory is concerned only with factors internal to the population, so nondeterminism reflects a lack of knowledge of the state of the entity (the entity being not any one particular organism but the population). Nondeterminism that arises through lack of knowledge concerning the state of the entity can be described by classical stochastic models (e.g. Markov processes) because the probability structure is Kolmogorovian (i the mathematical theory of natural selection. Selection theory is concerned only with factors internal to the population, so nondeterminism reflects a lack of knowledge of the state of the entity (the entity being not any one particular organism but the population). Nondeterminism that arises through lack of knowledge concerning the state of the entity can be described by classical stochastic models (e.g. Markov processes) because the probability structure is Kolmogorovian ( $i$ the mathematical theory of natural selection. Selection theory is concerned only with factors internal to the population, so nondeterminism reflects a lack of knowledge of the state of the entity (the entity being not any one particular organism but the population). Nondeterminism that arises through lack of knowledge 
concerning the state of the entity can be described by classical stochastic models (e.g. Markov processes) because the probability structure is Kolmogorovian (i the mathematical theory of natural selection. Selection theory is concerned only with factors internal to the population, so nondeterminism reflects a lack of knowledge of the state of the entity (the entity being not any one particular organism but the population). Nondeterminism that arises through lack of knowledge concerning the state of the entity can be described by classical stochastic models (e.g. Markov processes) because the probability structure is Kolmogorovian (i the mathematical theory of natural selection. Selection theory is concerned only with factors internal to the population, so nondeterminism reflects a lack of knowledge of the state of the entity (the entity being not any one particular organism but the population). Nondeterminism that arises through lack of knowledge concerning the state of the entity can be described by classical stochastic models (e.g. Markov processes) because the probability structure is Kolmogorovian (i the mathematical theory of natural selection. Selection theory is concerned only with factors internal to the population, so nondeterminism reflects a lack of knowledge of the state of the entity (the entity being not any one particular organism but the population 